\title{
Effect of the STAT3 inhibitor STX-0119 on the proliferation of a temozolomide-resistant glioblastoma cell line
}

\author{
TADASHI ASHIZAWA $^{1 *}$, YASUTO AKIYAMA ${ }^{1,3^{*}}$, HARUO MIYATA $^{1}$, AKIRA IIZUKA $^{1}$, \\ MASARU KOMIYAMA ${ }^{1}$, AKIKO KUME ${ }^{1}$, MAHO OMIYA ${ }^{1}$, TAKASHI SUGINO ${ }^{2}$, AKIRA ASAI $^{4}$, \\ NAKAMASA HAYASHI ${ }^{3}$, KOICHI MITSUYA $^{3}$, YOKO NAKASU ${ }^{3}$ and KEN YAMAGUCHI ${ }^{1}$ \\ ${ }^{1}$ Immunotherapy Division, Shizuoka Cancer Center Research Institute, Divisions of ${ }^{2}$ Pathology and ${ }^{3}$ Neurosurgery, \\ Shizuoka Cancer Center Hospital, Sunto-gun, Shizuoka 411-8777; ${ }^{4}$ Graduate School of Pharmaceutical Sciences, \\ University of Shizuoka, Suruga-ku, Shizuoka 422-8526, Japan
}

Received February 17, 2014; Accepted April 14, 2014

DOI: 10.3892/ijo.2014.2439

\begin{abstract}
Glioblastoma multiforme (GBM) is one of the most malignant and aggressive tumors and has a very poor prognosis, with a median survival time of less than 2 years. Once recurrence develops, there are few therapeutic approaches to control the growth of glioblastoma. In particular, temozolomide (TMZ)resistant (TMZ-R) GBM is very difficult to treat, and a novel approach to overcome resistance is eagerly awaited. Previously, we reported a novel small molecule inhibitor of STAT3 dimerization, STX-0119, as a cancer therapeutic. In the current study, the efficacy of STX-0119 was evaluated against our established TMZ-resistant U87 cell line using quantitative PCR-based gene expression analysis, in vitro assay and animal experiments. The growth inhibitory effect of STX-0119 on U87 and TMZ-R U87 cells was moderate $\left(\mathrm{IC}_{50}, 34\right.$ and $45 \mu \mathrm{M}$, respectively). In particular, STX-0119 did not show significant inhibition of U87 tumor growth; however, it suppressed the growth of the TMZ-R U87 tumor in nude mice by more than $50 \%$, and prolonged the median survival time compared to the control group. Quantitative PCR revealed that YKL-40, MAGEC1, MGMT, several EMT genes, mesenchymal genes and STAT3 target genes were upregulated, but most of those genes were downregulated by STX-0119 treatment. Furthermore, the invasive activity of TMZ-R U87 cells was
\end{abstract}

Correspondence to: Dr Yasuto Akiyama, Immunotherapy Division, Shizuoka Cancer Center Research Institute, 1007 Shimonagakubo, Nagaizumi-cho, Sunto-gun, Shizuoka 411-8777, Japan

E-mail: y.akiyama@scchr.jp

*Contributed equally

Abbreviations: GBM, glioblastoma multiforme; TMZ, temozolomide; MGMT, $\mathrm{O}^{6}$-methylguanine-DNA-methyltransferase; STAT, signal transducer and activator of transcription; shRNA, small hairpin RNA

Key words: signal transducer and activator of transcription 3 inhibitor, YKL-40, temozolomide resistance, glioblastoma significantly inhibited by STX-0119. YKL-40 levels in TMZ-R U87 cells and their supernatants were significantly decreased by STX-0119 administration. These results suggest that STX-0119 is an efficient therapeutic to overcome TMZ resistance in recurrent GBM tumors, and could be the next promising compound leading to survival prolongation, and YKL-40 may be a possible surrogate marker for STAT3 targeting.

\section{Introduction}

The signal transducer and activator of transcription (STAT) 3 is a point of convergence for various oncogenic signaling pathways through protein tyrosine kinases and is constitutively activated both in tumor cells and immune cells $(1,2)$. Furthermore, STAT3 activation promotes the production of immunosuppressive factors mainly by the tumor itself that induce immunoregulatory immune cells and a marked immunosuppressive environment around the tumor tissue $(3,4)$.

Glioblastoma multiforme (GBM) is one of the most malignant and aggressive tumors and has a very poor prognosis, with a mean survival time of less than 2 years even with the recent development of temozolomide (TMZ)-based intensive treatment $(5,6)$. Once recurrence develops, there are few therapeutic approaches to control the growth of glioblastoma. Therefore, in particular, TMZ-resistant GBM is very difficult to treat, and a novel approach to overcome resistance is eagerly awaited.

The activation of several signaling pathways, including receptor tyrosine kinase (7), Akt (8), MAPK (9), Wnt (10) and Notch and Hedgehog (11) pathways, is involved in the progression of GBM. Importantly, constitutive activation of the Janus kinase (JAK)/signal transducer and activator of transcription (STAT) pathway contributes to tumor progression by promoting cell proliferation and inhibiting apoptosis.

$\mathrm{O}^{6}$-methylguanine-DNA-methyltransferase (MGMT) is well known to remove methylation from the $\mathrm{O}^{6}$ position of guanine and contribute to TMZ resistance induction (12). It is generally accepted that high MGMT expression through the methylation of MGMT promoter is one of the mechanisms responsible for TMZ resistance. 
On the other hand, it is increasingly suggested that a mechanism other than MGMT can trigger TMZ resistance based on multiomics analysis. Several novel biomarkers linked to MGMT expression and the methylation status such as the HOX signature and EGFR expression (13), somatic mutation of mismatch repair gene MSH6 (14), prolyl 4-hydroxylase, $\beta$-polypeptide (P4HB), EGFR mutation (EGFRvIII) (15) and CD74, have been reported.

Considering that STAT3 might be another target for chemo-resistance treatment, several therapeutic agents, including small molecules acting as STAT3 activity inhibitors, have been reported to show antitumor effects through the control of chemo-resistance. Previously, we identified a novel inhibitor of STAT3 dimerization, STX-0119, which exhibited a potent antitumor effect on a human lymphoma cell line with highly activated STAT3 and GB-stem cell lines (16). In the present study, we investigated the effect of STX-0119 on a TMZ-resistant glioblastoma cell line in vitro and in vivo, and demonstrated that STX-0119 molecule significantly inhibited the proliferation of even a TMZ-resistant cell line and prolonged the survival of tumor-transplanted mice.

\section{Materials and methods}

Establishment of TMZ-resistant U87 cell line. U87 cells were purchased from the American Type Culture Collection (ATCC, Manassas, VA) and cultured in Dulbecco's modified Eagle's medium (DMEM) (Sigma-Aldrich, St. Louis, MO) supplemented with $10 \%$ fetal bovine serum (FBS; Life Technology, Carlsbad, CA), penicillin and streptomycin. The U87 parental cell line, which is sensitive to TMZ, was first maintained at a low dose of TMZ $(5 \mu \mathrm{M})$ and then successively exposed to incremental doses of TMZ (up to $150 \mu \mathrm{M}$ ). After killing the majority of cells, surviving cells were maintained until a normal rate of growth was obtained. The $\mathrm{IC}_{50}$ of $\mathrm{TMZ}$ was evaluated by the WST-1 assay. TMZ-resistant (R) U87 cells with more than 20 passages were maintained at a dose of $100 \mu \mathrm{M} \mathrm{TMZ}$ and used for in vitro and in vivo experiments.

Antibodies and reagents. Antibodies against STAT3, phosphospecific STAT3 (Tyr705), cleaved caspase-3, MGMT and $\beta$-actin were purchased from Cell Signaling Technology, Inc. (Danvers, MA) and Becton-Dickinson (BD) Biosciences (Franklin Lakes, NJ) for western blot analysis (WB). Mouse anti-human YKL-40 antibody was purchased from Abcam (Cambridge, MA, USA).

Chemicals. STX-0119 was supplied by the Center for Drug Discovery, University of Shizuoka (Shizuoka, Japan). These compounds were suspended and diluted in a sterile $0.5 \mathrm{w} / \mathrm{v} \%$ methyl cellulose $400 \mathrm{cp}$ solution (Wako, Tokyo, Japan) for use in animal experiments. TMZ was purchased from Sigma-Aldrich.

Cell proliferation assay. Cell proliferation was examined using the WST-1 assay (Dojin Kagaku Corp., Kumamoto, Japan) described previously (16). Briefly, $1 \times 10^{4}$ parental U87 or TMZ-R U87 cells were seeded into each well of a 96-well micro-culture plate (Corning Inc., Corning, NY) and compounds ranging from 0.25 to $500 \mu \mathrm{M}$ were added. After 4 days, the WST-1 substrate was added to the culture and optical density (OD) was measured at 450 and $620 \mathrm{~nm}$ using an immunoreader (Immuno Mini NJ-2300; Nalge Nunc International, Roskilde, Denmark). The $\mathrm{IC}_{50}$ value was defined as the dose needed for a $50 \%$ reduction in OD calculated from the survival curve. Percent survival was calculated as follows: (mean OD of test wells - mean OD of background wells)/(mean OD of control wells - mean OD of background wells) x100.

Cell invasion assay. The invasion assay using TMZ-R U87 cells was performed on Matrigel-coated $(0.33 \mathrm{mg} / \mathrm{ml})$ Transwell inserts with $8 \mu \mathrm{m}$ pore size (BD Biosciences). A total of $500 \mu \mathrm{l}$ cells at $2 \times 10^{4} / \mathrm{ml}$ were added to Transwells in triplicate, and $750 \mu \mathrm{l}$ DMEM containing $10 \%$ FBS was added to the lower wells. After 12-18 h incubation, cells that passed through the membrane were fixed and stained with Diff-Quik II solution (Siemens AG, Erlangen, Germany). Migrating cells were counted under a microscope. To analyze the effect of STX-0119 on invasion activity, TMZ-R U87 cells pre-treated with various doses of STX-0119 $(0,25,50$ and $100 \mu \mathrm{M}$ ) for $48 \mathrm{~h}$ were utilized in the assay.

Quantitative polymerase chain reaction ( $q P C R)$ analysis. Real-time PCR analysis of stem cell and neuronal markers, and STAT3 target genes using the 7500 Real-Time PCR System (Applied Biosystems, Foster, CA) was performed as described previously. Briefly, all PCR primers (CD24, YKL-40, GDF15, HLA-DQA1, MAGEC1, MGMT, MMP1 as TMZ-R U87 cell-specific genes; ALDH1A1, EGFR, GFAP, NANOG, NES, Oct3/4, SOX2 as GB stem cell markers; FN1, FOXC2, MMP2, SNAIL1, SNAIL2, TCF4, TWIST1, SMAD2 as EMT-associated genes; STAT3, FOSL2, C/EBP as GB mesenchymal marker gene; BCL2, Survivin, c-Myc, CXCL10, TGFB1, P53, HIF-1 $\alpha$ as STAT3 target genes) and TaqMan probes were purchased from Applied Biosystems. Parental U87 or TMZ-R U87 cells were treated with STX-0119 or DMSO for $24 \mathrm{~h}$, and total RNA was extracted. Complementary DNA was synthesized from $100 \mathrm{ng}$ total RNA and quantitative PCR was carried using a TaqMan RNA-to-Ct 1-Step kit (Applied Biosystems).

ELISA for human YKL-40. YKL-40 levels in the supernatant of parental U87 or TMZ-R U87 cells treated with STX-0119 were measured using human YKL-40-specific ELISA. Cells were plated in 96-well microplates (Corning) at $4 \times 10^{4}$ cells (200 $\mu \mathrm{l}$ cells at $\left.2 \times 10^{5} / \mathrm{ml}\right)$ per well. After cells were treated with STX-0119, WP1066 or DMSO for $24 \mathrm{~h}$, supernatants were collected and YKL-40 levels were measured.

Western blot analysis (WB). TMZ-R U87 cells were treated with STX-0119 or DMSO at various doses for $24 \mathrm{~h}$. Cells were lysed using RIPA buffer (Thermo Fisher Scientific Inc., Rockford, IL) containing protease inhibitors and phosphatase inhibitors and used for western blot analysis as described previously. Briefly, cell lysate was subjected to SDS-PAGE with a $7.5 \%$ polyacrylamide separating gel, and then transferred to PVDF membranes. After blocking, the membranes were incubated at $4^{\circ} \mathrm{C}$ overnight with the primary antibody against STAT3, phosphospecific STAT3, YKL-40 and $\beta$-actin $(1: 200-1: 2,000)$ in blocking solution. 

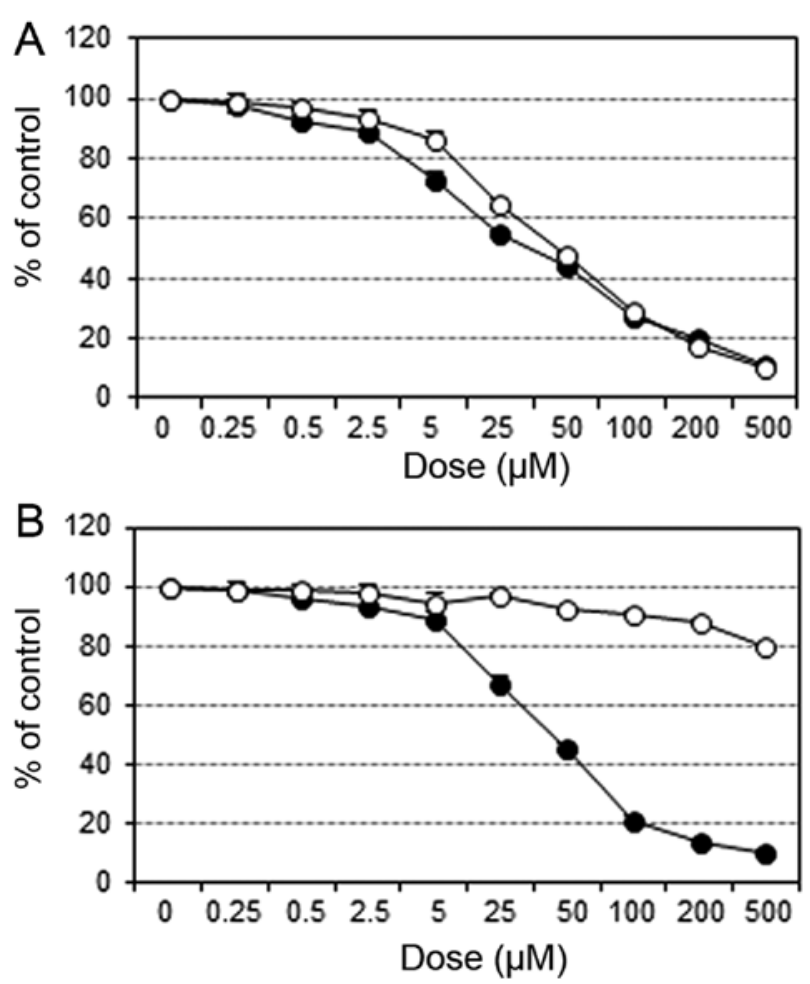

Figure 1. Inhibitory effect of STX-0119 on the proliferation of parental and TMZ-R U87 cell lines. (A) Parental U87 cell line, and (B) TMZ-R U87 cell line. (०) TMZ, (•) STX-0119. Each point shows the mean of triplicate samples.

After washing, the membranes were incubated for $1 \mathrm{~h}$ with horseradish peroxidase (HRP)-conjugated anti-mouse IgG (1:5,000). Membranes were treated with ECL plus reagent (GE Healthcare, Piscataway, $\mathrm{NJ}$ ) and analyzed on a chemiluminescence scanner (LAS-3000; Fujifilm, Tokyo, Japan). Apoptosis induction in TMZ-R U87 cells treated with STX-0119 for $24 \mathrm{~h}$ was investigated using a Caspase-3 Western detection kit including the primary antibody against cleaved caspase-3 (Cell Signaling).

Animal experiments. Male nude mice (BALB/cA-nu/nu, 5-6 weeks old) were obtained from Nippon Clea (Tokyo, Japan). All animals were cared for and used humanely according to the guidelines for the welfare and use of animals in cancer research, and the procedures were approved by the Animal Care and Use Committee of Shizuoka Cancer Center Research Institute.

Parental U87 cells $\left(1 \times 10^{6}\right)$ and TMZ-R U87 cells $\left(1 \times 10^{6}\right)$ were inoculated into the flank of BALB/cA-nu/nu mice. To evaluate the antitumor activity against subcutaneous (s.c.) inoculated tumors, tumor volume was calculated based on the National Cancer Institute formula as follows: tumor volume $\left(\mathrm{mm}^{3}\right)=$ length $(\mathrm{mm}) \times$ [width $\left.(\mathrm{mm})\right]^{2} \times 1 / 2$.

STX-0119 at doses of 40 and $80 \mathrm{mg} / \mathrm{kg}$ was administered orally daily from day 0 to day 4 followed by 2 days of rest, which was repeated three times over 21 days. TMZ at a dose of $5 \mathrm{mg} / \mathrm{kg}$ was administered orally daily from day 0 to day 4 . The efficacy of compounds against human tumor cells inoculated into nude mice was expressed as the mean $V / V_{0}$ value and evaluated as reported previously (16).

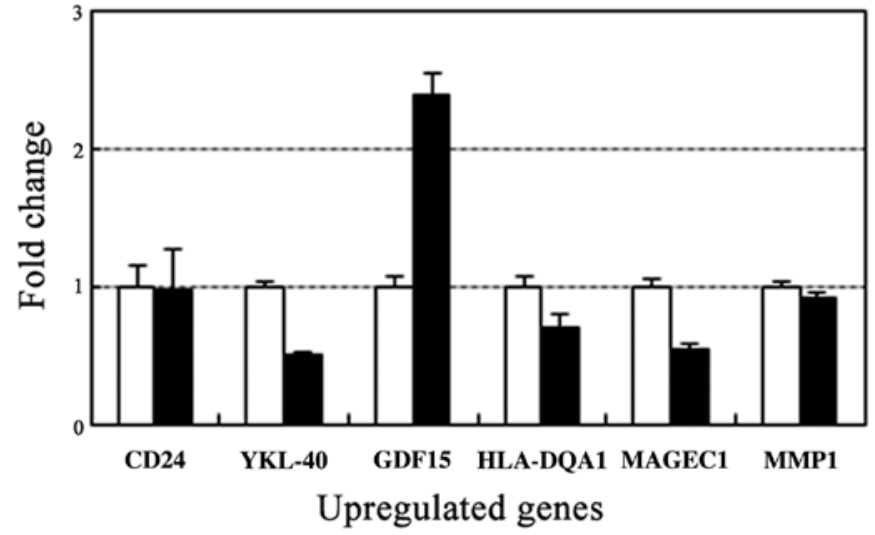

Figure 2. Effect of STX-0119 on the expression of upregulated genes in TMZ-R U87 cell line. TMZ-R U87 cell line was treated with $100 \mu \mathrm{M}$ STX-0119 for $24 \mathrm{~h}$. The gene expression level without STX-0119 was rated 1 as a control, and gene expression in STX-0119-treated TMZ-R U87 cells was shown as a fold increase using real-time PCR. Each column shows the mean \pm SD of four samples. Open column, control; closed column, treated with STX-0119.

Statistical analysis. Statistical difference was analyzed using Student's t-test. Values of $\mathrm{P}<0.05$ were considered statistically significant. For the in vivo experiment, statistical analysis was performed with corrected P-values to compare with the untreated control using Mann-Whitney rank-sum test, and to determine the effect of compounds on survival of tumor-bearing mice the log-rank test was used for Kaplan-Meier survival curves.

\section{Results}

Cell proliferation assay. The growth inhibitory effects of STX-0119 and TMZ on parental U87 cells were similar (STX-0119 $\mathrm{IC}_{50}, 34 \mu \mathrm{M}$; TMZ IC $50,45 \mu \mathrm{M}$ for U87 cells). Whereas, the effect of STX-0119 on TNZ-R U87 was not so different from U87 cells; however, TMZ had no inhibitory effect on TMZ-R U87 cells (STX-0119 $\mathrm{IC}_{50}, 43 \mu \mathrm{M}$; $\mathrm{TMZ} \mathrm{IC}_{50},>500 \mu \mathrm{M}$ for TMZ-R U87 cells) (Fig. 1).

Effect of STX-0119 on upregulated genes specific for TMZ-R U87 cell line. Based on differential gene expression profiling (unpublished data) between parental U87 and TMZ-R U87 cells, the upregulated genes specific for TMZ-R U87 cells were CD24, YKL-40, GDF15, HLA-DQA1, MAGEC1, MMP1 and MGMT were upregulated more than 10-fold and 5-fold, respectively, in TMZ-R U87 cells compared to parental U87 cells using real-time PCR. YKL-40 and MAGEC1 gene expressions were significantly decreased after treatment with STX-0119 at $100 \mu \mathrm{M}$ (Fig. 2).

Effect of STX-0119 on STAT3 and YKL-40 protein expressions in TMZ-R U87 cell line. The activation (phosphorylation) of STAT3 and upregulation of YKL-40 were identified in the TMZ-R U87 cell line compared to the U87 cell line (data not shown). YKL-40 mRNA was decreased by STX-0119 in a dose-dependent manner. YKL-40 protein was markedly downregulated in $100 \mu \mathrm{M}$ STX-0119-treated cells, and even in the supernatant. However, the impact of STX-0119 on STAT3 and phosphorylated STAT3 expression was marginal after treatment. 

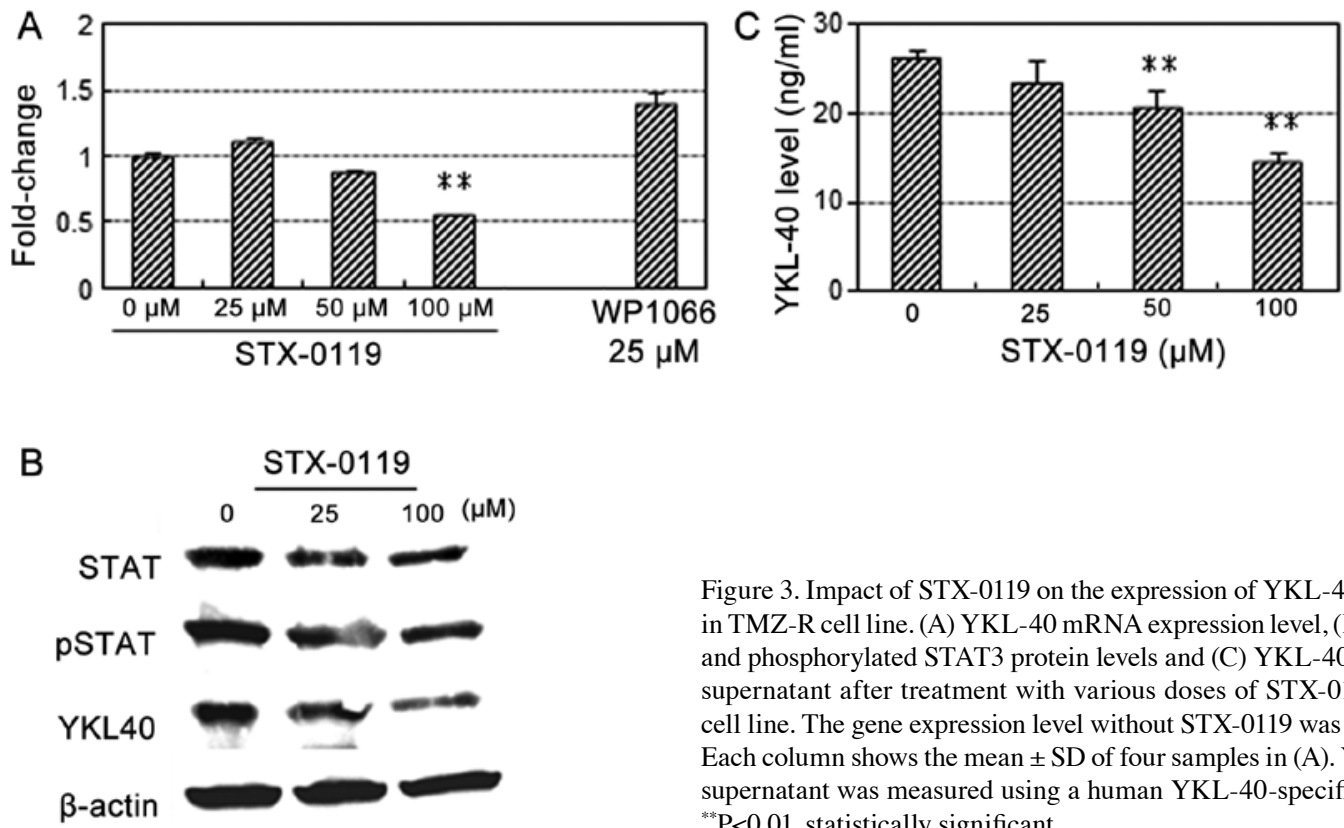

Figure 3. Impact of STX-0119 on the expression of YKL-40 and STAT3 genes in TMZ-R cell line. (A) YKL-40 mRNA expression level, (B) YKL-40, STAT3 and phosphorylated STAT3 protein levels and (C) YKL-40 protein level in the supernatant after treatment with various doses of STX-0119 in TMZ-R U87 cell line. The gene expression level without STX-0119 was rated 1 as a control. Each column shows the mean \pm SD of four samples in (A). YKL-40 level in the supernatant was measured using a human YKL-40-specific ELISA kit in (C). ${ }^{* *} \mathrm{P}<0.01$, statistically significant.

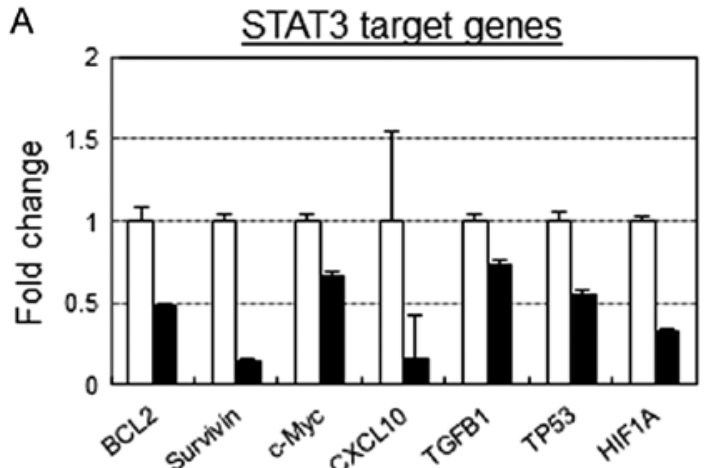

Genes

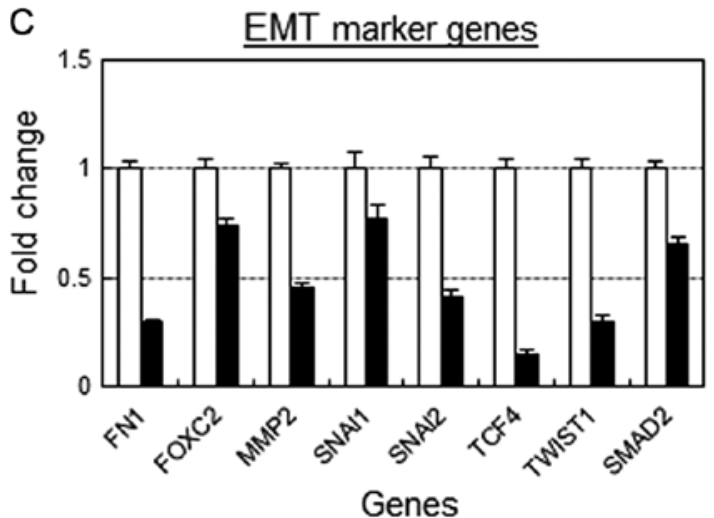

B

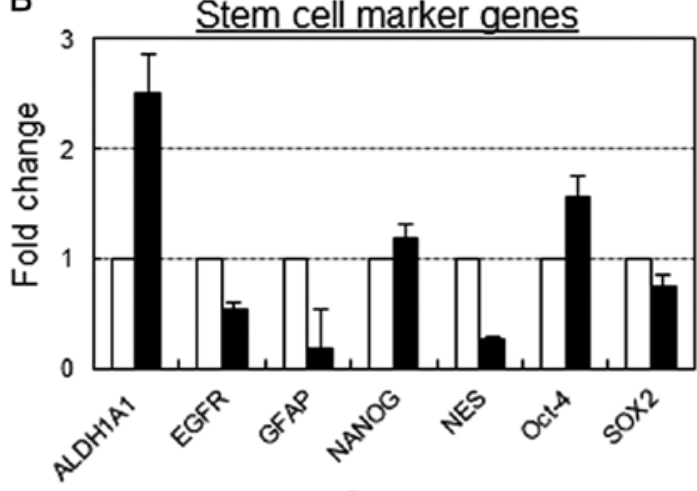

Genes

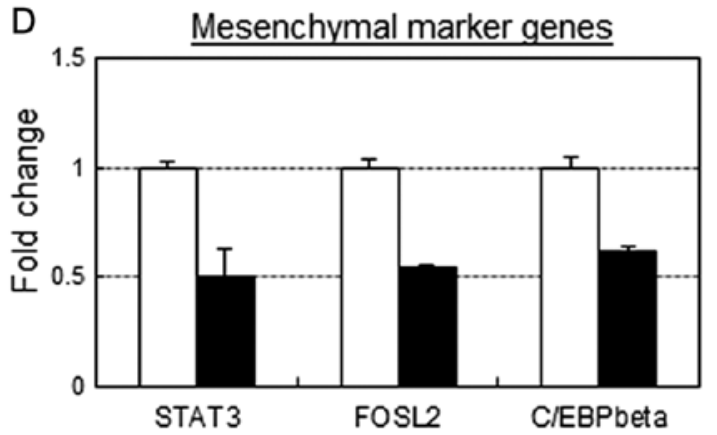

Genes

Figure 4. Effect of STX-0119 on the expression of various mRNA genes of TMZ-R U87 cell line. (A) STAT3 target genes, (B) stem cell marker genes, (C) EMT marker genes and (D) mesenchymal marker genes. TMZ-R U87 cell line was treated with $100 \mu \mathrm{M}$ STX-0119 for $24 \mathrm{~h}$. The mRNA expression level without STX-0119 was rated 1 as a control, and gene expression in STX-0119-treated TMZ-R U87 cells was shown as a fold increase using real-time PCR. Each column shows the mean \pm SD of four samples. Open column, control; closed column, treated with STX-0119.

Effect of STX-0119 on upregulated genes from various marker groups in TMZ-R U87 cell line. Real-time PCR analysis demonstrated that many marker gene expressions were upregulated more than 2-fold in TMZ-R U87 cells compared to parental
U87 cells as follows: BCL2, Survivin, c-Myc, CXCL10, TGFB1, P53, HIF-1 $\alpha$ as STAT3 target genes; ALDH1A1, EGFR, GFAP, NANOG, NES, Oct3/4, SOX2 as GB stem cell markers; FN1, FOXC2, MMP2, SNAIL1, SNAIL2, TCF4, TWIST1, SMAD2 


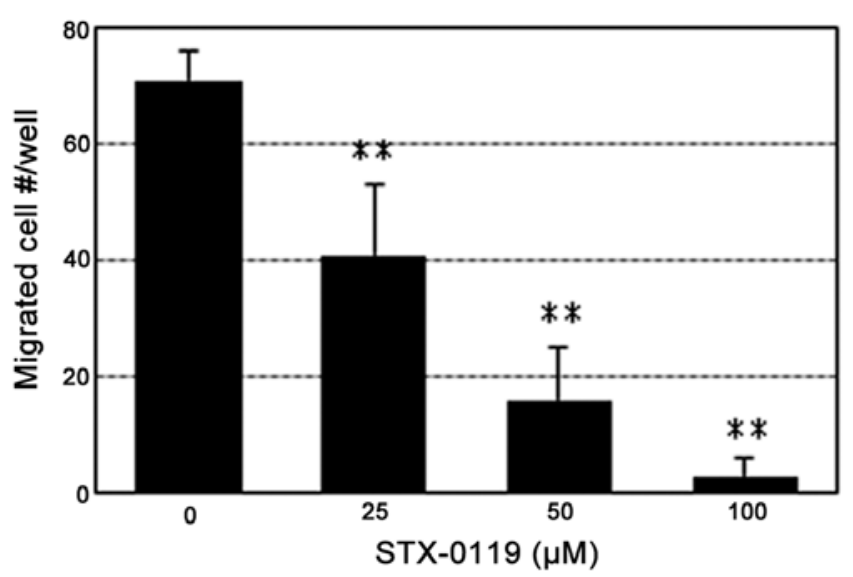

Figure 5. Inhibition of the invasion activity of TMZ-R U87 cell line by STX-0119. TMZ-R U87 cell line was treated with various doses of STX-0119 for $48 \mathrm{~h}$ and then used for the invasion assay. The migrated cell number was significantly suppressed after treatment with STX-0119 in a dose-dependent manner. Each column shows the mean $\pm \mathrm{SD}$ of three samples. ${ }^{* *} \mathrm{P}<0.01$.

as EMT-associated genes; STAT3, FOSL2, C/EBP as GB mesenchymal marker gene (data not shown). Several genes, including BCL2, Survivin, CXCL10, HIF1A, GFAP, NES, FN1, MMP2, SNAI2, TCF4 TWIST1, showed decreased expression by STX-0119 (Fig. 4A-C). Additionally, the effect of STX-0119 on mesenchymal markers was moderate (Fig. 4D).

Impact of STX-0119 on invasion activity of TMZ-R U87 cells. TMZ-R U87 cells were shown to possess greater invasion activity than parental U87 (data not shown). The invasion activity of TMZ-R U87 cells was reduced STX-0119 dose-dependently. Pre-treatment of TMZ-R U87 cells with STX-0119 at $100 \mu \mathrm{M}$ suppressed invasion activity by more than $90 \%$ compared to the without compound (Fig. 5).

Apoptosis induction by STX-0119 in TMZ-R U87 cell line. With regard to the cleaved caspase- 3 level, its expression increased at $>50 \mu \mathrm{M}$ STX-0119. TMZ-R U87 cells treated with STX-0119 at $100 \mu \mathrm{M}$ demonstrated an increase of the cleaved caspase-3 expression in TMZ-R U87 cells (Fig. 6).

STX-0119 inhibits tumor growth of TMZ-R U87 cells in vivo. TMZ-R U87 cell-transplanted mice showed significant resistance to TMZ and a shorter survival time in vivo, while parental U87 cell-transplanted mice exhibited obvious sensitivity to TMZ. The inhibitory effect of STX-0119 on parental U87 cells was marginal and did not show any survival benefit compared to the control. In contrast, the growth inhibition by TMZ on U87 cells was marked and showed no tumor recurrence until day 42 and obvious prolongation of survival (Fig. 7, Table I).

On the other hand, STX-0119 demonstrated a greater growth-inhibitory effect on TMZ-R U87 cells with more than 50\% inhibition compared to the control. Additionally, STX-0119 showed an obvious and more efficient prolongation of survival in TMZ-R U87-bearing mice compared to the TMZ group, which exhibited no growth inhibition. These effects of STX-0119 were identified in the $40 \mathrm{mg} / \mathrm{kg}$ group,

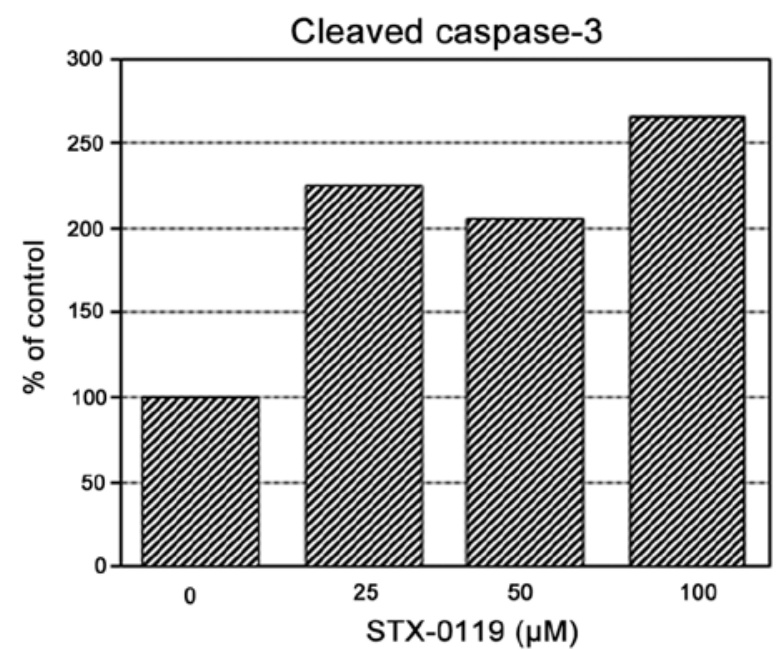

Cleaved caspase-3

$\beta$-actin

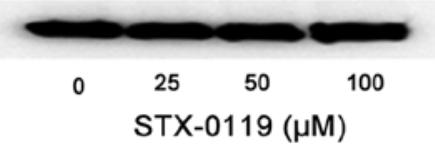

Figure 6. Apoptosis induction in TMZ-R U87 cells treated with STX-0119. TMZ-R U87 cell line was treated with various doses of STX-0119 for $24 \mathrm{~h}$ and used for WB analysis of cleaved caspase-3. The protein expression level without STX-0119 was rated as 100\% for the control. Each column shows the mean of two experiments.

but not at $80 \mathrm{mg} / \mathrm{kg}$ (Fig. 7, Table I). In addition, STX-0119 showed no adverse effects on tumor-bearing mice.

\section{Discussion}

High-grade gliomas including glioblastoma multiforme (GBM) are the most malignant and aggressive of tumors, and have a very poor prognosis and a high recurrence rate, with a mean survival time of less than 2 years even with the recent development of an intensive temozolomide (TMZ)based treatment protocol. Additionally, frequent recurrence even after chemo-radiation treatment is a crucial problem in the clinical field, which should be overcome to extend the overall survival of GBM.

$\mathrm{O}^{6}$-methylguanine-DNA-methyltransferase (MGMT) is well known to remove methylation from the $\mathrm{O}^{6}$ position of guanine and contribute to TMZ resistance induction. Accumulated evidence demonstrated that high MGMT expression through the methylation of MGMT promoter is one of the mechanisms responsible for TMZ resistance. However, recently, several researchers demonstrated that major mechanisms other than MGMT are involved in TMZ resistance. Several biomarkers linking to MGMT expression and the methylation status, such as HOX signature and EGFR expression, somatic mutation of mismatch repair gene MSH6, prolyl 4-hydroxylase, $\beta$-polypeptide (P4HB), EGFR mutation (EGFRvIII) and CD74, have been demonstrated. With regard to novel approaches to overcome MGMT-related resistance to TMZ, bortezomib (BZ) as a proteasome inhibitor (17), and 


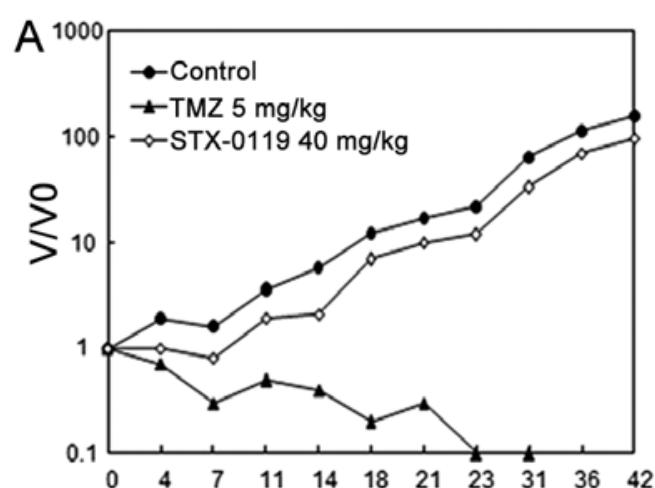

Days after initial administration

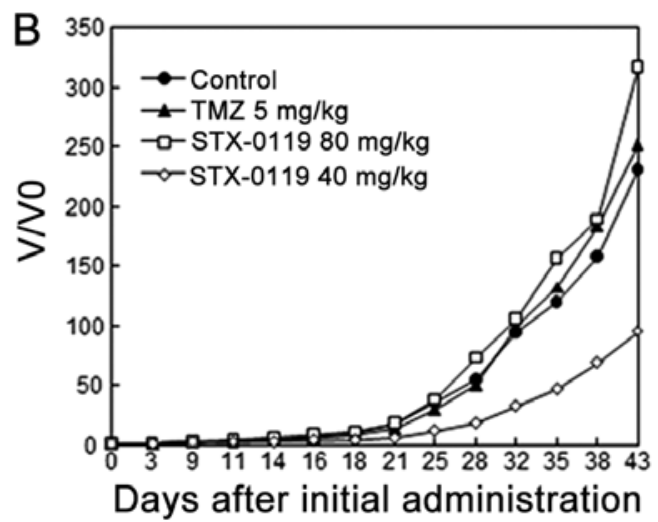

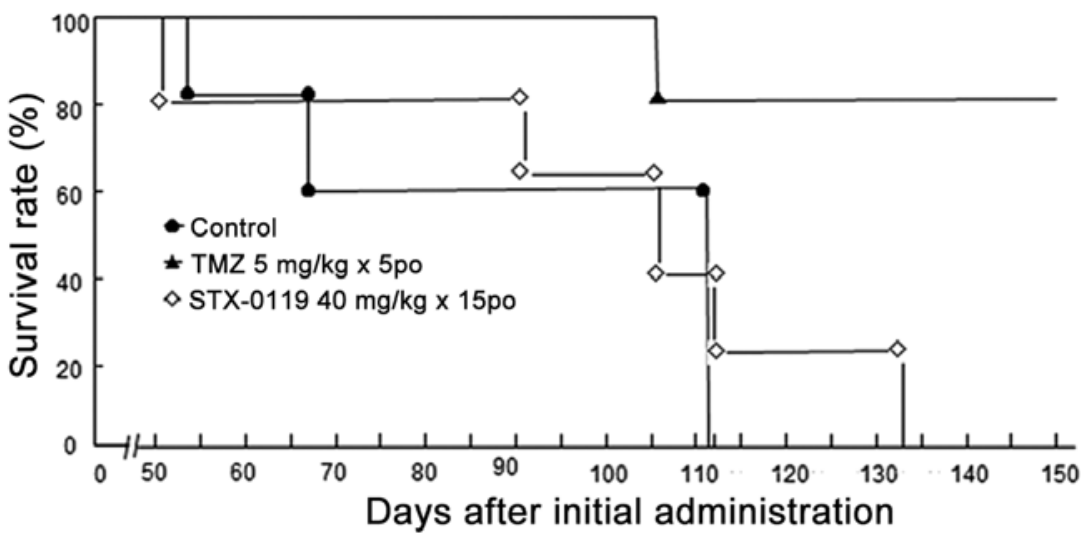

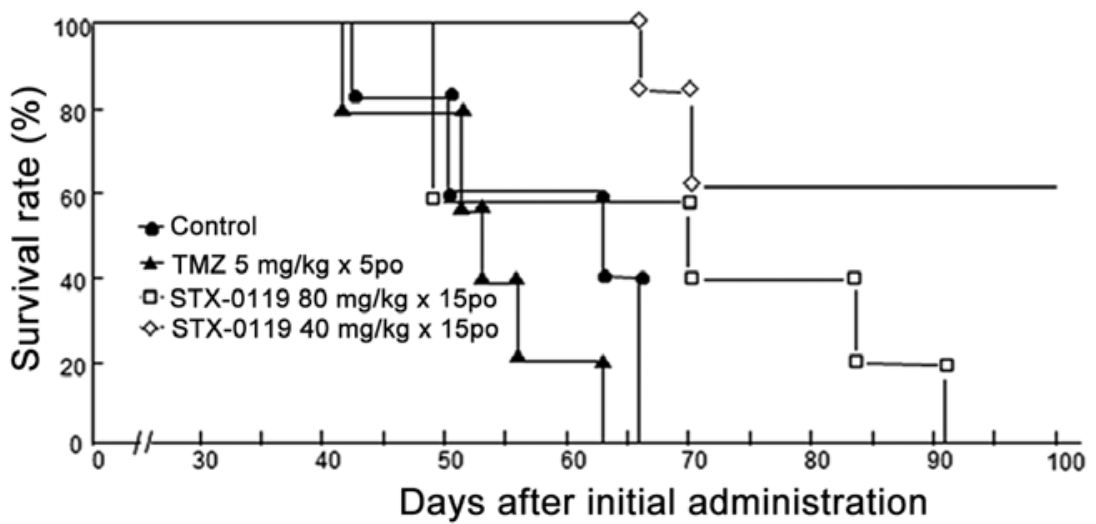

Figure 7. Inhibitory effect of STX-0119 on in vivo tumor growth of parental and TMZ-R U87 cells. Nude mice transplanted with (A) parental U87 and (B)

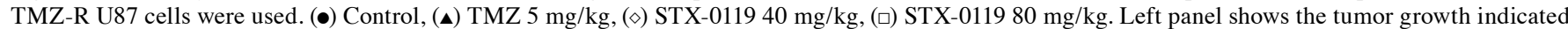
as $\mathrm{V} / \mathrm{V}_{0}$ value and the right panel shows the survival time of tumor-bearing mice treated with compounds. Each point shows the mean value of 5 mice.

Table I. Survival analysis of U87 parental and TMZ-R cell-derived tumors.

\begin{tabular}{|c|c|c|c|c|c|}
\hline Compound & $\begin{array}{c}\text { Dose } \\
(\mathrm{mg} / \mathrm{kg})\end{array}$ & Frequency & $\begin{array}{c}\text { Mean survival } \\
\text { (days) }\end{array}$ & $\begin{array}{l}\text { ILS } \\
(\%)\end{array}$ & $\begin{array}{l}\text { Tumor-free } \\
\text { at } 150 \text { days }\end{array}$ \\
\hline \multicolumn{6}{|c|}{ U87 parental cell tumor } \\
\hline Control & & & $91.4 \pm 28.6$ & 0 & $0 / 5$ \\
\hline TMZ & 5 & 5p.o. QD5 & $>141.2 \pm 19.7$ & $>54$ & $4 / 5(\mathrm{P}=0.026)$ \\
\hline STX-0119 & 40 & 15p.o. (QD5/wk) & $98.6 \pm 30.6$ & 8 & $0 / 5(\mathrm{P}=0.934)$ \\
\hline \multicolumn{6}{|c|}{ U87 TMZ-R cell tumor } \\
\hline Control & & & $57.6 \pm 10.7$ & 0 & $0 / 5$ \\
\hline TMZ & 5 & 5p.o. QD5 & $53.0 \pm 7.6$ & -8 & $0 / 5$ \\
\hline STX-0119 & 40 & 15p.o. (QD5/wk) & $87.2 \pm 17.6$ & 51 & $3 / 5(\mathrm{P}=0.021)$ \\
\hline STX-0119 & 80 & 15p.o. (QD5/wk) & $68.6 \pm 19.4$ & 19 & $0 / 5(\mathrm{P}=0.145)$ \\
\hline
\end{tabular}

even inactivation of MGMT by gene therapy (18) have been demonstrated to show moderate effects on MGMT downregulation and tumor cell death.

On the other hand, a significant association between STAT3 signaling and GBM or GBM stem cell development and maintenance has been demonstrated in recent studies (19-23), which emphasizes the potential of STAT3 as a therapeutic target. Some studies demonstrated that STAT3 is required for the proliferation and maintenance of multipotency in GBM stem cells through siRNA-mediated STAT3 inhibition (19).
Previously, we reported that STX-0119, a novel small molecule inhibitor of STAT3 dimerization, showed antitumor activity in vitro and in vivo against a highly STAT3 activated lymphoma cell line and primary GBM stem cells (24). In particular, STX-0119 showed a growth-suppressive effect on even highly TMZ-resistant GBM stem cells derived from a recurrent GB tumor. With regard to the correlation of STAT3 and TMZ resistance, novel observations have been reported. Kohsaka et al (25) reported that STAT3 inhibition downregulated MGMT expression, and there was a significant 
positive correlation between the expression level of MGMT and phosphorylated STAT3 in high-grade glioma tumors. Villalva et al (26) demonstrated that a STAT3 inhibitor, Stattic-mediated STAT3 inhibition, sensitized GBM stem cells to the inhibitory action of TMZ with a synergistic effect. Interestingly, intratumoral hypoxia might be a promoting factor localized in immature GBM stem cells with high MGMT expression, with strong resistance to TMZ and STAT3 activation in the inner core of the tumor mass (27). This observation suggested that a combination of TMZ and HIF-1 inhibitors may have additional antitumor effects against GBM stem cells with TMZ resistance. Another approach to overcoming TMZ resistance is the combination of TMZ and interferon (IFN)- $\beta$, which is supposed to show a synergistic antitumor effect against TMZ-resistant GBM cell lines, such as T98G cells, through the downregulation of MGMT and STAT3 $(28,29)$. Motomura et al (30) reported the benefits of IFN- $\beta$ and TMZ combination therapy for newly diagnosed GBM patients in a multicentered clinical trial; the median survival time (MST) of the combination therapy patients was significantly greater than the TMZ-alone group (19.9 versus 12.7 months). STAT3 inhibitor compounds including AG490, WP1066 and Curcumin have been reported to exhibit antitumor activity through suppression of the invasive and migratory activity of malignant glioma cells (31-33). In particular, WP1066 was shown to reduce intratumoral JAK2/ STAT3 activity and prolong the survival of animals given a GBM stem cell xenograft. However, these compounds have not yet been reported to show antitumor activity against TMZ-resistant GBM tumors.

Gene expression profiling, which can predict the response to TMZ in high-grade gliomas, has also been performed. Yoshino et al (34) reported that differential gene sets identified using a DNA microarray between TMZ-sensitive and -resistant GBM cell lines were not causal factors in the TMZ response besides MGMT. Our similar analysis using a DNA microarray between TMZ-sensitive and -resistant U87 cells demonstrated that YKL-40 and MAGEC1 genes, besides MGMT, were identified as TMZ-resistance-specific genes (unpublished data). The precise mechanism of YKL-40 involvement in TMZ resistance is yet to be clarified; however, considering the downregulation of YKL-40 expression after STX-0119 treatment, YKL-40 might be a possible surrogate marker for STAT3 targeting TMZ-R glioblastoma.

A recent study using next-generation genome sequencing for recurrent glioma after TMZ treatment demonstrated that newly identified gene mutations in the recurrent tumor, probably induced by TMZ, might be driver mutations promoting tumor growth (35). This result strongly suggests that novel mutations are new causal factors involved in the TMZ resistance mechanism in recurrent glioma after TMZ therapy.

Finally, in the current study, we established a solid TMZ-resistant U87 cell line which showed obvious resistance in an in vivo model and demonstrated that STX-0119 showed a potent antitumor effect on TMZ-resistant tumors where STX-0119 significantly prolonged MST in tumor-bearing mice. These results suggest that STX-0119 is a potential therapeutic to overcome TMZ resistance in recurrent GBM tumors, and could be the next promising compound leading to survival prolongation. This is the first report that a STAT3 inhibitor small molecule compound clearly showed an anti-tumor effect against TMZ-resistant GBM cells. Based on our new observations regarding the efficacy mechanism of STX-0119, more efficient compound development is expected.

\section{Acknowledgements}

This study was supported by a grant from a regional innovation strategy support program of the Ministry of Education, Culture, Sports, Science and Technology, Japan.

\section{References}

1. Zhong Z, Wen L and Darnell JE Jr: Stat3 and Stat4: members of the family of signal transducers and activators of transcription. Proc Natl Acad Sci USA 91: 4806-4810, 1994.

2. Bromberg J and Darnell JE Jr: The role of STATs in transcriptional control and their impact on cellular functions. Oncogene 19: 2468-2473, 2000.

3. Yu H, Kortylewski M and Pardoll D: Crosstalk between cancer and immune cells: role of STAT3 in the tumor microenvironment. Nat Rev Immunol 7: 41-51, 2007.

4. Wang T, Niu G, Kortylewski M, et al: Regulation of the innate and adaptive immune responses by Stat-3 signaling in tumor cells. Nat Med 10: 48-54, 2004.

5. Stupp R, Mason WP, van den Bent MJ, et al: Radiotherapy plus concomitant and adjuvant temozolomide for glioblastoma. N Engl J Med 352: 987-996, 2005.

6. Mirimanoff RO, Gorlia T, Mason W, et al: Radiotherapy and temozolomide for newly diagnosed glioblastoma: recursive partitioning analysis of the EORTC 26981/22981-NCIC CE3 phase III randomized trial. J Clin Oncol 24: 2563-2569, 2006.

7. Brennan C, Momota H, Hambardzumyan D, Ozawa T, Tandon A, Pedraza A and Holland E: Glioblastoma subclasses can be defined by activity among signal transduction pathways and associated genomic alterations. PLoS One 4: e7752, 2009.

8. Cheng CK, Fan QW and Weiss WA: PI3K signaling in gliomaanimal models and therapeutic challenges. Brain Pathol 19: 112-120, 2009.

9. Demuth T, Reavie LB, Rennert JL, et al: MAP-ing glioma invasion: mitogen-activated protein kinase 3 and p38 drive glioma invasion and progression and predict patient survival. Mol Cancer Ther 6: 1212-1222, 2007.

10. Pu P, Zhang Z, Kang C, Jiang R, Jia Z, Wang G and Jiang H: Downregulation of Wnt 2 and beta-catenin by siRNA suppresses malignant glioma cell growth. Cancer Gene Ther 16: 351-361, 2009.

11. Purow BW, Haque RM, Noel MW, et al: Expression of Notch-1 and its ligands, Delta-like-1 and Jagged-1, is critical for glioma cell survival and proliferation. Cancer Res 65: 2353-2363, 2005.

12. Hegi ME, Diserens AC, Gorlia T, et al: MGMT gene silencing and benefit from temozolomide in glioblastoma. $\mathrm{N}$ Engl $\mathbf{J}$ Med 352: 997-1003, 2005.

13. Murat A, Migliavacca E, Gorlia T, et al: Stem cell-related 'self-renewal' signature and high epidermal growth factor receptor expression associated with resistance to concomitant chemoradiotherapy in glioblastoma. J Clin Oncol 26: 3015-3024, 2008.

14. Hunter C, Smith R, Cahill DP, et al: A hypermutation phenotype and somatic MSH6 mutations in recurrent human malignant gliomas after alkylator chemotherapy. Cancer Res 66: 3987-3991, 2006.

15. Mukherjee B, McEllin B, Camacho CV, et al: EGFRvIII and DNA double-strand break repair: a molecular mechanism for radioresistance in glioblastoma. Cancer Res 69: 4252-4259, 2009.

16. Ashizawa T, Miyata H, Ishii H, et al: Antitumor activity of a novel small molecules STAT3 inhibitor against a human lymphoma cell line with high STAT3 activation. Int J Oncol 38: 1245-1252, 2011.

17. Vlachostergios PJ, Hatzidaki E, Befani CD, Liakos P and Papandreau CN: Bortezomib overcomes MGMT-related resistance of glioblastoma cell lines to temozolomide in a schedule-dependent manner. Invest New Drugs 31: 1169-1181, 2013. 
18. Jiang G, Wei ZP, Pei DS, Xin Y, Liu YQ and Zheng JN: A novel approach to overcome temozolomide resistance in glioma and melanoma: Inactivation of MGMT by gene therapy. Biochem Biophys Res Commun 406: 311-314, 2011.

19. Li GH, Wei H, Lv SQ, Ji H and Wang DL: Knockdown of STAT3 expression by RNAi suppresses growth and induces apoptosis and differentiation in glioblastoma stem cells. Int J Oncol 37: 103-110, 2010

20. See AP, Han JE, Phallen J, et al: The role of STAT3 activation in modulating the immune microenvironment of GBM. J Neurooncol 110: 359-368, 2012.

21. Sherry MM, Reeves A, Wu JK and Cochran BH: STAT3 is required for proliferation and maintenance of multipotency in glioblastoma stem cells. Stem Cells 27: 2383-2392, 2009.

22. Liu Y, Li C and Lin J: STAT3 as a therapeutic target for glioblastoma. Anticancer Agents Med Chem 10: 512-519, 2010.

23. Jackson C, Ruzevick J, Amin AG and Lim M: Potential role for STAT3 inhibitors in glioblastoma. Neurosurg Clin N Am 23: 379-389, 2012.

24. Ashizawa T, Miyata H, Iizuka A, et al: Effect of the STAT3 inhibitor STX-0119 on the proliferation of cancer stem-like cells derived from recurrent glioblastoma. Int J Oncol 43: 219-227, 2013

25. Kohsaka S, Wang L, Yachi K, et al: STAT3 inhibition overcomes temozolomide resistance in glioblastoma by donregulating MGMT expression. Mol Cancer Ther 11: 1289-1299, 2012.

26. Villalva C, Martin-Lanneree S, Cortes U, et al: STAT3 is essential for the maintenance of neurosphere-initiating tumor cells in patients with glioblastomas: a potential for targeted therapy? Int J Cancer 128: 826-838, 2011.

27. Pistollato F, Abbadi S, Rampazzo E, et al: Intratumoral hypoxic gradient drives stem cells distribution and MGMT expression in glioblastoma. Stem Cells 28: 851-862, 2010.
28. Yuki K, Natsume A, Yokoyama H, et al: Induction of oligodendrogenesis in glioblastoma-initiating cells by IFN-mediated activation of STAT3 signaling. Cancer Lett 284: 71-79, 2009.

29. Yoshino A, Ogino A, Yachi K, et al: Effect of IFN- $\beta$ on human glioma cell lines with temozolomide resistance. Int J Oncol 35: 139-148, 2009.

30. Motomura K, Natsume A, Kishida Y, et al: Benefits of interferon- $\beta$ and temozolomide combination therapy for newly diagnosed primary glioblastoma with the unmethylated MGMT promoter: A multicenter study. Cancer 117: 1721-1730, 2011.

31. Senft C, Priester M, Polacin M, Schroder K, Seifert V, Kogel D and Weissenberger J: Inhibition of the JAK-2/STAT3 signaling pathway impedes the migratory and invasive potential of human glioblastoma cells. J Neurooncol 101: 393-403, 2011.

32. Stechishin OD, Luchman HA, Ruan Y, et al: On-target JAK2/STAT3 inhibition slows disease progression in orthotopic xenografts of human glioblastoma brain tumor stem cells. Neuro Oncol 15: 198-207, 2013.

33. Senft C, Polacin M, Priester M, Seifert V, Kogel D and Weissenberger J: The nontoxic natural compound Curcumin exerts anti-proliferative, anti-migratory, and anti-invasive properties against malignant gliomas. BMC Cancer 10: 491, 2010.

34. Yoshino A, Ogino A, Yachi K, et al: Gene expression profiling predicts response to temozolomide in malignant gliomas. Int J Oncol 36: 1367-1377, 2010.

35. Johnson BE, Mazor T, Hong C, et al: Mutational analysis reveals the origin and therapy-driven evolution of recurrent glioma. Science 343: 189-193, 2013. 